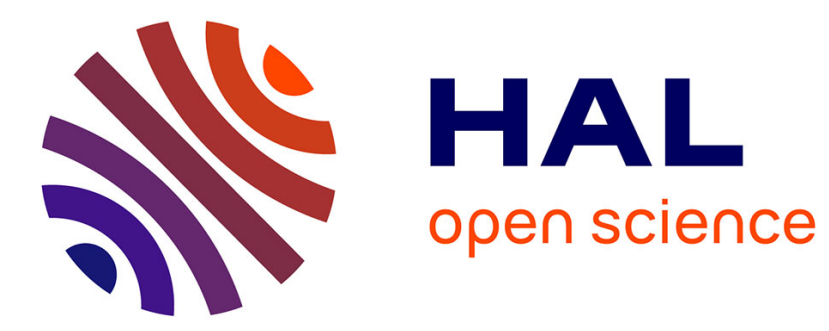

\title{
Permeability of a bubble assembly: From the very dry to the wet limit
}

Florence Rouyer, Olivier Pitois, Elise Lorenceau, N. Louvet

\section{To cite this version:}

Florence Rouyer, Olivier Pitois, Elise Lorenceau, N. Louvet. Permeability of a bubble assembly: From the very dry to the wet limit. Physics of Fluids, 2010, 22 (4), pp.043302. 10.1063/1.3364038 . hal-00507028

\section{HAL Id: hal-00507028 \\ https://hal.science/hal-00507028}

Submitted on 10 Sep 2015

HAL is a multi-disciplinary open access archive for the deposit and dissemination of scientific research documents, whether they are published or not. The documents may come from teaching and research institutions in France or abroad, or from public or private research centers.
L'archive ouverte pluridisciplinaire HAL, est destinée au dépôt et à la diffusion de documents scientifiques de niveau recherche, publiés ou non, émanant des établissements d'enseignement et de recherche français ou étrangers, des laboratoires publics ou privés. 


\title{
Permeability of a bubble assembly: From the very dry to the wet limit
}

\author{
Florence Rouyer, Olivier Pitois, Elise Lorenceau, and Nicolas Louvet \\ Laboratoire de Physique des Matériaux Divisés et des Interfaces, Université Paris-Est, \\ UMR CNRS 8108, 5 bvd Descartes, 77454 Marne la Vallée Cedex 2, France
}

(Received 10 April 2009; accepted 18 February 2010; published online 6 April 2010)

\begin{abstract}
Bubble assemblies offer the remarkable property of adjusting their packing fraction over three orders of magnitude, thus providing an interesting system for the study of liquid flows through granular matter. Although significant work has been done in several fields of research, e.g., foams, porous media, and suspensions, a complete set of data over such a wide range of porosity $\varepsilon$ is still lacking. In this paper, we measure the permeability of a bubbly system in the range $0.1<\varepsilon<0.8$ and we connect these new data with a recently published set obtained for foams corresponding to $\varepsilon<0.2$ [E. Lorenceau et al., Eur. Phys. J. E 28, 293 (2009)]. Moreover, measurements performed with two different surfactants, the so-called "mobile" and "nonmobile" interfaces, allow us to determine the influence of the bubbles' surface mobility, which is proved to be a significant parameter up to $\varepsilon \approx 0.6$, thus well above the bubbles packing fraction. Above $\varepsilon \approx 0.6$, surface elasticity is fully mobilized over the bubbles' surface and the behavior of rigid spheres is observed for both solutions. We show that all the permeability values obtained for the bubble assembly with "nonmobile" interfaces are properly described with the Carman-Kozeny model. () 2010 American Institute of Physics. [doi:10.1063/1.3364038]
\end{abstract}

\section{INTRODUCTION}

Dispersions of gas bubbles in liquid exhibit a large variety of dynamical behavior. It is a rich field of fundamental research, as bubbles assemblies are often used as model systems for soft matter. Moreover, bubbly systems are used in a lot of industrial applications: gas is mixed in many materials to improve their mechanical or acoustic properties or to make them lighter. In these bubbly systems, the homogeneity of the sample can be drastically affected by the drainage of the interstitial liquid (and the simultaneous rising of the bubbles); thus justifying the large amount of work devoted to the understanding of drainage in foams, for example. Note however that in spite of the significant progress realized in this field, most of the results only concern aqueous dry foams, ${ }^{1,2}$ whereas mostly loose bubbly systems are encountered in industry.

From a more general point of view, flows through bubbles assemblies are similar to flows in porous media made of packed beads and in settling suspensions of hard spheres. In these systems, the Reynolds number is generally low meaning that viscous effects are dominant compared to inertia. However, the interfacial mobility behavior induced by the surfactant of the foaming solution has to be taken into account in addition to the ability of bubbles to form thin films when packed. For foams, it has been shown that high interfacial mobility, the so-called "mobile" behavior, could increase significantly the velocity of the liquid flow at a given porosity, ${ }^{3}$ which definitely distinguishes this "soft" system from its "solid" counterpart. Very recently however, the permeability of foams characterized by less mobile interfaces, the so-called "nonmobile" behavior, was found to be properly described by the model of Carman-Kozeny, ${ }^{4}$ originally validated for packed beds of solid spheres, i.e., liquid volume fractions (porosity) $\varepsilon \approx 0.4$. Note, however that the influence of the mobility parameter is nontrivial: while for dry foams $(\varepsilon \approx 0.01)$ this effect was found to govern the drainage dynamics, for wet foams $(\varepsilon \approx 0.1)$ this influence seems to be reduced. ${ }^{3}$ This complex behavior might be due to the loss of confinement as the porosity increases or to the behavior of the foam films, which are believed to have a significant effect on the flow through foam channels, ${ }^{5}$ despite the small quantity of liquid they comprise. Yet, one has to stress that published data for foam permeability do not allow concluding for the influence of the mobility parameter above $\varepsilon \approx 0.1-0.2$. This raises the following major question of the influence of the surfactant used to stabilize the bubbly system close to the packing fraction, i.e., when foam films disappear. The complexity of such system might also remain above the packing fraction: as the liquid content increases, the typical size of the interstices varies and the boundary conditions for the flow at the surface of the bubbles are expected to evolve as well. The present paper aims to bring an experimental answer to this tricky and open issue. In contrast to previous works performed on aqueous foams, the foam permeability is measured over a very large range for the porosity, $0.1<\varepsilon<0.8$, thanks to a dedicated fluidized bed method. The main interest of this new set of data is that, for the first time, the bubble packing transition range is covered, for both "mobile" and "nonmobile" systems. Moreover, we connect these new data with a recently published set obtained for foams, i.e., $\varepsilon<0.2{ }^{3}$ allowing for complete curves to be plotted over three orders of magnitude for the porosity. In the discussion of the paper, it is shown that the data can be described in the range $0.6<\varepsilon<0.8$ by models dedicated to non-Brownian hard spheres suspensions for both "mobile" and "nonmobile" systems. All the permeability values obtained for the bubble assembly with "nonmobile" interfaces are properly described with the Carman-Kozeny model. 


\section{FLUIDIZED BED EXPERIMENT}

We build up a fluidized bed of monodisperse bubbles and measure its permeability over a large range of porosity. Soap solution is used to stabilize the contacts between bubbles and thus to avoid coalescence. To investigate the influence of the boundary conditions for the liquid flow at the surface of the bubbles, we use two different soap solutions known for providing opposite interfacial behaviors. ${ }^{3}$

\section{A. Materials}

As explained above, we use two soap solutions. In order to connect the results obtained in this work with available data for foams permeability, we chose the same solutions than those used in Ref. 3. A solution of tetradecyltrimethylammoniumbromide (TTAB) at a concentration of $3 \mathrm{~g} / \mathrm{l}$ was found to give the highest permeability values in the range of $\varepsilon<0.1-0.2{ }^{3}$ Interfaces obtained with this solution will be referred to as "mobile" interfaces. In opposition, a solution of TTAB $3 \mathrm{~g} / \mathrm{l}$ mixed with $0.2 \mathrm{~g} / \mathrm{l}$ dodecanol was found to give the smallest permeability values within the same range for the porosity. ${ }^{3}$ The corresponding interfaces will be referred to as "nonmobile" interfaces. For both solutions, surfactant concentration is well above the critical micelle concentration $(\mathrm{CMC}=1 \mathrm{~g} / \mathrm{l})$, the density is $\rho=1000 \mathrm{~kg} / \mathrm{m}^{3}$, the bulk shear viscosity is $\eta=1 \mathrm{mPa}$ s. For "mobile" and "nonmobile" interfaces, respectively, surface tensions are $\gamma=38$ and $25 \mathrm{mN} / \mathrm{m}$, and surface shear viscosities $\eta_{s} \sim 10^{-8}$ and $10^{-6} \mathrm{~kg} \mathrm{~s}^{-1}$.

The gas used to create the bubbles is saturated with perfluorohexane $\left(\mathrm{C}_{6} \mathrm{~F}_{14}\right)$ to avoid size evolution of the bubbles during the experiment.

\section{B. Experimental setup}

The fluidized bed is constructed in a Plexiglas column (radius $R=4.75 \mathrm{~mm}$ and height $=200 \mathrm{~mm}$ ), set vertically and partially immersed in a bath of the soap solution. A needle is placed at the open bottom of the column and allows monodisperse bubbles to be produced at constant gas flow rate $Q_{g}$ (cf. Fig. 1). The top of the column ends with a grid (gray in Fig. 1) and is connected to a syringe pump that delivers soap solution at fixed liquid flow rate $Q_{l}$ in the range of $0.01-10 \mathrm{ml} / \mathrm{min}$. The mesh size of the grid is smaller than the bubble diameter. This latter has been chosen such that the Reynolds number Re for the liquid flow through the bubbles assembly is small. For "mobile" and "nonmobile" interfaces, respectively, the bubble size is $D=260$ and $190 \mu \mathrm{m}$. The maximum value of $\mathrm{Re}$ is obtained at high porosity, for which $\operatorname{Re}=\rho V_{\text {Stokes }} D / \eta \approx 4$ for bubbles of $200 \mu \mathrm{m}$ in diameter ( $V_{\text {Stokes }}$ is the Stokes velocity equal to the terminal velocity of an isolated buoyant sphere in an infinite medium with no slip conditions on its surface at low Re). We checked that the bubble bed was stable during measurements for every liquid fraction. Note also that the ratio $R / D$ is larger than 20 , which prevent from pertubative wall effects and that the Bond number is of the order of $10^{-3}$, meaning that bubbles are expected to remain spherical for porosity above packing. ${ }^{7}$

Initially, a small liquid flow rate $\left(Q_{l}<1 \mathrm{ml} / \mathrm{min}\right)$ is

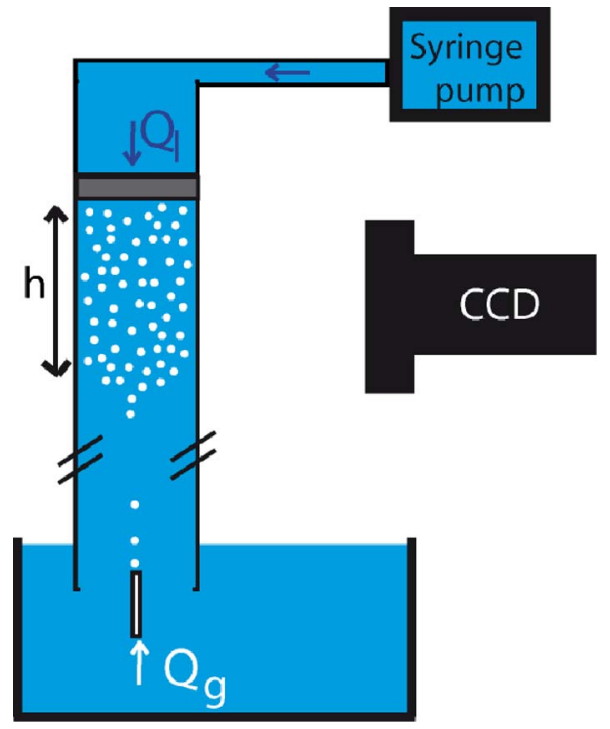

FIG. 1. (Color online) Sketch of the experimental setup of the fluidized bed.

imposed from top to bottom of the column while the gas bubbles are produced with at a rate $Q_{g}$ at the bottom of the column. The resulting growth of the bubble assembly is characterized by the rate $d h / d t$, where $h$ is the bubble assembly height (see Fig. 1). The evolution $h(t)$ is followed with a charge coupled device camera, allowing for $d h / d t$ to be measured. Note that we checked that $d h / d t$ is constant during the bed production. Then, the gas flow rate is stopped for $h>3 R$, and the corresponding initial height is noted $h_{i}$. Next, the bubble bed is fully fluidized by a large liquid flow rate $\left(Q_{l}>5 \mathrm{ml} / \mathrm{min}\right)$. Finally, several liquid flow rates are successively imposed and the corresponding height $h$ is measured. The initial porosity $\varepsilon_{i}$ and the porosity $\varepsilon$ of the bubble bed for each liquid flow rate are, respectively, calculated with the following equations:

$$
\varepsilon_{i}=1-\frac{Q_{g}}{\left(\pi R^{2}\right) \frac{d h}{d t}} \quad \text { and } \quad \varepsilon=1-\left(1-\varepsilon_{i}\right) \frac{h_{i}}{h} .
$$

\section{Permeability measurements}

The permeability of the bubble assembly is deduced from the Darcy law ${ }^{8}$ with a pressure gradient induced by buoyancy $^{9,10}$

$$
k=\frac{\mu Q_{l}}{\Delta \rho g \pi R^{2}(1-\varepsilon)}=\frac{1}{18} \frac{Q_{l}}{V_{\text {Stokes }} \pi R^{2}(1-\varepsilon)} D^{2} .
$$

\section{RESULTS AND DISCUSSION}

Normalized permeability curves $k / D^{2}$ are shown in Fig. 2 as a function of porosity for both soap solutions. Data already published for foams ${ }^{3}(\varepsilon<0.1-0.2)$ are also presented to be compared to the present work. First of all, it is shown that this new set of data connects well with the previous one, although both the measurement method and the setup are different. To this respect, permeability values ob- 


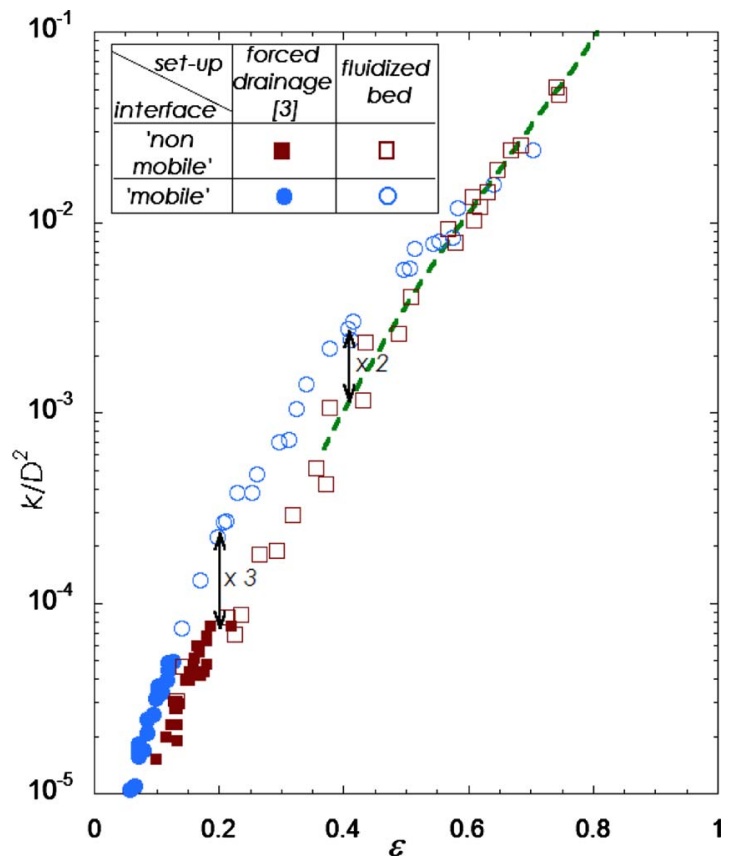

FIG. 2. (Color online) Permeability of particulate systems made of monodisperse bubbles as a function of porosity. Experimental data of the fluidized bed are compared with previous experimental data of foam (see legend for details). The dotted line corresponds to permeability calculated by $k / D^{2}$ $=1 / 18\left[\varepsilon^{4.9} /(1-\varepsilon)\right]$, which is derived for hard spheres suspensions (Ref. 18).

tained in this work are in agreement with the data deduced from previous experiments with the forced drainage method in the range of $0.1<\varepsilon<0.2$. We recall that this latter method is known to be problematic for liquid fractions above 0.1 due to the appearance of convective instabilities. ${ }^{11}$ Second, the present measurement method allowed for the permeability to be plotted over three decades for a unique porous system. Note that the corresponding range of porosity has been increased by a factor of 4 with respect to published data.

Now, we focus on data obtained at high porosity, i.e., $\varepsilon>0.6$. As observed in Fig. 2, the two sets of data overlap in this range of porosity, suggesting that the boundary conditions are similar for both systems. In other words, the mobility parameter introduced for the study of foam drainage has no influence as the liquid fraction reaches 0.6 . This can be understood considering the work performed on isolated bubbles rising in soap solutions. Indeed, the rising velocity is close to the velocity of buoyant spheres with no slip conditions due to a kinetic rigidification induced by Marangoni flows at the surface. ${ }^{12,13}$ It is thus deduced that (i) this mechanism affects both systems of "mobile" and "nonmobile" interfaces, and (ii) the rigidification effect predicted for isolated bubbles covered with surfactants appears as soon as the liquid fraction raises close to 0.6 in the bubble assembly. Consequently, the corresponding permeability is expected to be described by models derived for non-Brownian hard sphere suspensions. Up to now, the Richardson-Zaki (RZ) empirical law is the reference for average settling velocity of suspensions, ${ }^{14}$ and it is also valid to describe the upward fluid velocity in case of fluidized suspensions ${ }^{15}$ this law writes $V_{\text {settling }}=V_{\text {Stokes }} \varepsilon^{5.5},{ }^{16}$ thus $k / D^{2}=1 / 18\left[\varepsilon^{5.5} /(1-\varepsilon)\right]$. More recently, using a mean field approach to estimate the energy dissipation during the homogeneous sedimentation or the particulate fluidization of non-Brownian hard spheres in a concentrated suspension, Mills and Snabre retrieved the RZ fit in the limit of low Reynolds number ${ }^{17}$ and proposed to account for inertial effects in it. In their model, the average settling velocity of suspensions is $V_{\text {settling }}=V_{\text {Stokes }} \varepsilon^{n}$, where $n$ is a decreasing function of the particulate Reynolds number $\left(\operatorname{Re}_{\mathrm{p}}\right) \cdot{ }^{18}$ In particular, $n=4.9$ for $\mathrm{Re}_{\mathrm{p}}=4$. We see in Fig. 2 that the theoretical prediction $k / D^{2}=1 / 18\left[\varepsilon^{4.9} /(1-\varepsilon)\right]$ describes very well the experimental data at large porosity $(\varepsilon>0.6)$. The maximum relative deviation is less than $10 \%$ for both solutions. We conclude that indeed, for moderate $\mathrm{Re}_{\mathrm{p}}$ and small Bo, bubble assemblies stabilized with surfactants behave like hard sphere suspensions for $\varepsilon>0.6$.

We now discuss the data at intermediate porosities $0.2<\varepsilon<0.6$, around the packing fraction $\varepsilon \approx 0.4$. The permeability behavior is expected to be complex because the permanent foam films existing in the bubble assembly for $\varepsilon<0.4$ disappear for $\varepsilon>0.4$. Note also that transient foam films may survive due to bubbles collisions.

First we consider the case of the "nonmobile" interfaces. In this system, we have shown that the boundary conditions resemble the ones for rigid interfaces for $\varepsilon>0.6$. Besides, a recent work indicated that "nonmobile" interfaces really mimic rigid walls at the scale of the bubbles for $\varepsilon<0.2{ }^{4} \mathrm{In}$ order to investigate the interfaces behavior in the intermediate range of porosity, we use the Carman-Kozeny model, that has been proved to be relevant for the description of permeability with this system for $\varepsilon<0.2{ }^{4}$ We recall that the model relates the specific surface area $S$ to the permeability of a porous medium characterized by a porosity $\varepsilon$ (Ref. 19)

$$
k=\frac{\varepsilon^{3}}{5 S^{2}} .
$$

Physically, the specific surface area represents the inverse of the characteristic length of channel that constitutes the porous medium, and thus it is the inverse of the relevant length for estimating viscous dissipation, i.e., the mean hydraulic radius: $m=\varepsilon / S$. Originally, this model has been developed for granular beds. ${ }^{20}$ Very recently, the model was adapted to foams, considering the effective porosity induced by capillary effects; ${ }^{4}$ the bubbles are not spherical and each bubble shares a film (two very close interfaces) with its neighbors. The specific surface area has been estimated from the geometrical Kelvin cells models, for foam porosities $0.001<\varepsilon<0.32$ [packing fraction of the Kelvin (bcc) arrangement of spheres]. Note that remarkably, the constant "5," which was proposed by Carman for beds of rigid grains around $\varepsilon \approx 0.36$ was found to be in full agreement with permeability measurements for foams within the full investigated range of liquid fraction. For $\varepsilon>0.32$, bubbles are mainly spherical and the specific surface area is equal to $S=6(1-\varepsilon) / D$, similarly to bed and suspension of rigid spheres. The inset of Fig. 3 represents the variation of the normalized specific surface area SD as a function of $\varepsilon$ for foams, i.e., $0<\varepsilon<0.32$ (from Ref. 4), and hard spheres suspensions, i.e., $0.36<\varepsilon<1$. Note that at small porosity $(\varepsilon<0.05), S$ varies as the square root of $\varepsilon: S(\varepsilon) D \approx 15.8 \sqrt{\varepsilon}$. 


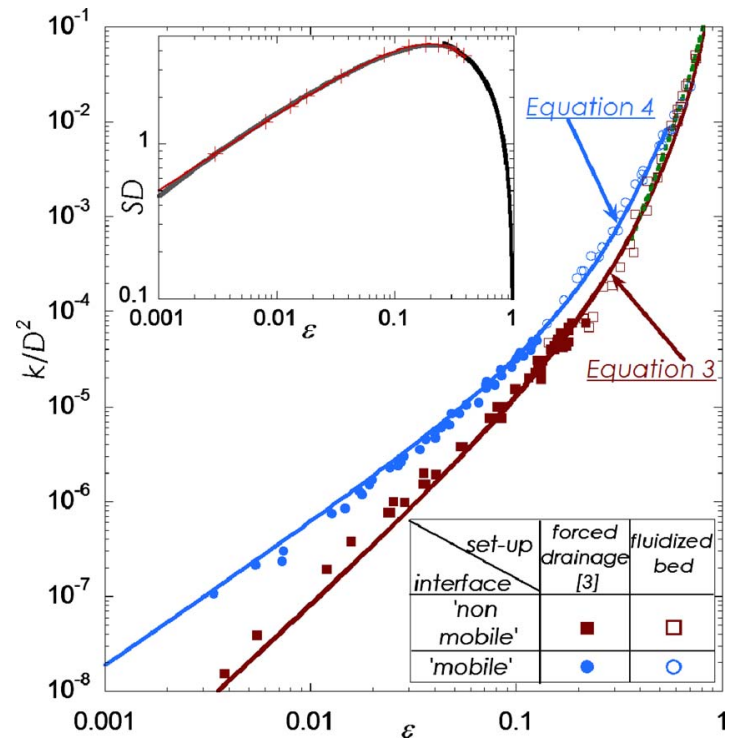

FIG. 3. (Color online) Permeability of particulate systems made of monodisperse bubbles as a function of porosity. Experimental data (see legend for details) are compared with the permeability calculated from CarmanKozeny law [Eq. (3)] and to fit proposed in Eq. (4). Inset: Variation in the normalized specific surface area of particulate systems made of monodisperse entities as a function of porosity. For $\varepsilon<0.32$ (lighter line) the specific surface of foam as explained in the text (Ref. 4). For $\varepsilon>0.32$ (black) $S D$ $=\pi D^{3} n=6(1-\varepsilon)$. The plus symbol line corresponds to the function $S(\varepsilon) D$ $\approx 15.8 \sqrt{\varepsilon}\left(1-2.15 \varepsilon+1.37 \varepsilon^{2}\right)$.

SD reaches a maximal value (approximately 4 ) for porosity around 0.2. To conveniently describe the variation in $S$ for $0<\varepsilon<0.4$, we propose to introduce an empirical function $g(\varepsilon)$ such as $S(\varepsilon) D \approx 15.8 \sqrt{\varepsilon} g(\varepsilon)$. This function should be chosen in such a way that it satisfies the limit conditions $g(0)=1$ and insures the continuity of the function $S(\varepsilon)$ for $\varepsilon=0.4$, i.e., $S(0.4) D=3.6$ and $d S / d \varepsilon(0.4) D=-6$. Assuming that $g$ is a function of second order, we find $g(\varepsilon)=1-2.15 \varepsilon$ $+1.37 \varepsilon^{2}$. The specific surface is thus describes as $S(\varepsilon) D$ $\approx 15.8 \sqrt{\varepsilon} g(\varepsilon)$ for $\varepsilon<0.4$ and $S(\varepsilon) D=6(1-\varepsilon)$ for $\varepsilon>0.4$. We note that the function $g(\varepsilon)$ is not a fit but rather a basic function that permits us to connect the description at low and high porosity. From the above, the theoretical permeability is

$$
\begin{aligned}
& k(\varepsilon)=\frac{\varepsilon^{2}}{1248\left(1-2.15 \varepsilon+1.37 \varepsilon^{2}\right)^{2}} D^{2} \quad \text { for } \varepsilon<0.4, \\
& k(\varepsilon)=\frac{\varepsilon^{3}}{180(1-\varepsilon)^{2}} D^{2} \quad \text { for } \varepsilon>0.4 .
\end{aligned}
$$

It is shown in Fig. 3 that Eq. (3) describes very well previous and present results corresponding to the system with nonmobile interfaces over the whole range explored for the porosity. This law permits us to describe experimental results from low to high porosity which is not trivial has recently shown for emulsion by Peron et al. ${ }^{21}$ At this point, we conclude that the bubble assembly made of the called "nonmobile" interface really mimics porous media made of no-slip solid walls over the whole range of porosity. Note also that over the large range $0.4<\varepsilon<0.85$, CarmanKozeny model provides permeability values in fair agreement with the model for hard spheres suspensions (the maxi- mum relative deviation is less than 20\%). Eventually, we stress that in the fluidized bed the particles are in permanent agitation due to hydrodynamic interactions, ${ }^{22,23}$ but the present result suggests that the effects induced by agitation of the fluidized bed are not crucial for permeability. This experimental observation is consistent with previous numerical simulations, ${ }^{9}$ but differs from Davies et al. conclusions, who stated that the Carman-Kozeny constant $c$ can be much larger than 5 and depend on porosity as well as on particle suspension properties. ${ }^{10}$ Here, we demonstrate that a unique constant allows to describe fairly well the permeability of suspensions made of monodisperse spherical entities (solid particles or bubbles with "nonmobile" interface).

Finally, we discuss the results for the system with "mobile" interfaces in the intermediate porosity range. For convenience, we also provide an analytical expression for this system (see Fig. 3)

$$
k(\varepsilon)=\frac{\varepsilon^{3 / 2}}{1700\left(1-2.7 \varepsilon+2.2 \varepsilon^{2}\right)^{2}} D^{2} \quad \text { for } \varepsilon<0.4,
$$

$$
k(\varepsilon)=\frac{\varepsilon^{3}}{180(1-\varepsilon)^{2}} D^{2} \quad \text { for } \varepsilon>0.6 .
$$

There is not yet a model to predict permeability for the "mobile" interfaces. However, we can guess that the length scale over which viscous dissipation would occur is smaller than the length scale associated to specific surface area in the Kozeny-Carman model. In particular, it would decrease as the mobility increases.

The data for the system of "mobile" interfaces are found to be larger than for "nonmobile" interfaces. Yet, previous results ${ }^{3}$ suggested an overlapping expected for liquid fractions close to 0.2. It is shown here that around the packing porosity $(\varepsilon \approx 0.4)$, the permeability of the system with mobile interfaces is approximately twice the permeability of the system with nonmobile interfaces. This deviation, remaining between 0.4 and 0.6, is rather surprising as Marangoni stresses are expected to rigidify the surface of rising bubbles in both soap solutions. On the other hand, it has been shown that for dry foams, the bubbles' surface is not rigidified by Marangoni stresses. This behavior is not fully understood but seems to be related to the presence of the foam films. ${ }^{5}$ The mobilization of surface elasticity (and the resulting surface rigidification) is therefore expected to be correlated with the proportion of those films in the bubble assembly. However, the present result indicates that the full rigidification of the bubbles' surface do not coincide with the vanishing of films at the packing fraction, as highlighted by the two arrows in Fig. 2. Instead, the surface rigidification develops progressively in the intermediate porosity range, i.e., $0.2<\varepsilon<0.6$. This unexpected behavior could be attributed to the complex coupling of surfactant surface flows and liquid flow between the bubbles. Although such mechanism has been considered 
for isolated rising bubbles, more complex situations involving several bubbles has never been investigated. To this respect, the present work provides an interesting experimental result to be elucidated.

\section{CONCLUSION}

We present experimental data for the permeability of a bubble assembly over a wide porosity range $(0.15-0.75)$ with two different surfactants, characterized by their opposite interfacial rheological behavior, the so-called "mobile" and "nonmobile" interfaces. These new data connect well with previous one obtained for foams, i.e., the dry limit, and for non-Brownian hard spheres suspensions, i.e., the wet limit. We prove that the bubbles' surface mobility is a significant parameter up to $\varepsilon \approx 0.6$. Indeed, the surface rigidification develops progressively in the intermediate porosity range, i.e., $0.2<\varepsilon<0.6$, instead of being a sharp event around packing when soap films shrink to zero. This unexpected behavior, which could be attributed to the complex coupling of surfactant surface flows and liquid flow between the bubbles, remains to be elucidated.

For the particular case of "nonmobile" interfaces, we show that the Carman-Kozeny law properly accounts for the evolution of permeability as a function of liquid fraction. More precisely, we demonstrate that a unique constant $(c$ $=5$ ), proposed initially by Carman for beds of rigid grains around $\varepsilon \approx 0.36$ allows to describe fairly well the permeability of bubbly assemblies over almost three orders of magnitude.

We give fits to estimate the variation in permeability as function of porosity from 0 to 0.85 for both "mobile" and "nonmobile" interfaces. We assume that these functions envelop the permeability curves as a function of porosity for any assembly of monodisperse spherical entities for porosity varied from 0 up to 0.85 .

We expect that this work is of great interest for other systems, in addition to foam and suspensions, where the continuous liquid phase varies over a wide range and the rheological properties of the interfaces between phases might be complex, for example, emulsions or complex biological systems.

\section{ACKNOWLEDGMENTS}

We thank Pierre Mills and Jerome Martin for fruitful discussions. We thank Jeffrey Morris for valuable comments. We gratefully acknowledge financial support from Agence Nationale de la Recherche (Contract No. ANR-05-JCJC0234-01), E.S.A. (MAP No. A099-108: C14914/02/NL/SH), and the French Space Agency (Convention No. CNES/ 70980).
${ }^{1}$ D. Weaire, N. Pittet, S. Hutzler, and D. Pardal, "Steady-state drainage of an aqueous foam," Phys. Rev. Lett. 71, 2670 (1993); G. Verbist, D. Weaire, and A. M. Kraynik, "The foam drainage equation," J. Phys.: Condens. Matter 8, 3715 (1996).

${ }^{2}$ S. A. Koehler, S. Hilgenfeldt, and H. A. Stone, "A generalized view of foam drainage: Experiment and theory," Langmuir 16, 6327 (2000).

${ }^{3}$ E. Lorenceau, N. Louvet, F. Rouyer, and O. Pitois, "Permeability of aqueous foams," Eur. Phys. J. E 28, 293 (2009).

${ }^{4}$ O. Pitois, N. Louvet, E. Lorenceau, and F. Rouyer, "Specific surface area model for foam permeability," Langmuir 25, 97 (2009).

${ }^{5}$ O. Pitois, N. Louvet, and F. Rouyer, "Recirculation model for liquid flow in foam channels," Eur. Phys. J. E 30, 27 (2009).

${ }^{6}$ O. Pitois, C. Fritz, and M. Vignes-Adler, "Liquid drainage through aqueous foam: Study of the flow on the bubble scale," J. Colloid Interface Sci. 282, 458 (2005).

${ }^{7}$ S. G. Yiantsios and R. H. Davis, "Close approach and deformation of two viscous drops due to gravity and van der Waals forces," J. Colloid Interface Sci. 144, 412 (1991); M. H. Manga and H. A. Stone, "Buoyancydriven interactions between two deformable viscous drops," J. Fluid Mech. 256, 647 (1993).

${ }^{8} \mathrm{H}$. Darcy, Les Fontaines Publiques de la Ville de Dijon (V. Dalmont, Paris, 1856).

${ }^{9}$ A. J. C. Ladd, "Hydrodynamic transport coefficients of random dispersions," J. Chem. Phys. 93, 3484 (1990); R. J. Hill, D. L. Koch, and A. J. C. Ladd, "The first effects of fluid inertia on flows in ordered and random arrays of spheres," J. Fluid Mech. 448, 213 (2001).

${ }^{10}$ L. Davies and D. Dollimore, "Theoretical and experimental values for the parameter $\mathrm{k}$ of the Kozeny-Carman equation, as applied to sedimenting suspensions," J. Phys. D 13, 2013 (1980).

${ }^{11}$ S. Hutzler, D. Weaire, and R. Crawford, "Convective instability in foam drainage," Europhys. Lett. 41, 461 (1998).

${ }^{12}$ V. G. Levich, Physicochemical Hydrodynamics (Prentice-Hall, Englewood Cliffs, NJ, 1962).

${ }^{13} \mathrm{~F}$. Takemura, "Adsorption of surfactants onto the surface of a spherical rising bubble and its effect on the terminal velocity of the bubble," Phys. Fluids 17, 048104 (2005); B. Cuenot, J. Magnaudet, and B. Spennato, "The effects of slightly soluble surfactants on the flow around a spherical bubble," J. Fluid Mech. 339, 25 (1997).

${ }^{14} \mathrm{X}$. Yin and D. L. Koch, "Hindered settling velocity and microstructure in suspensions of solid spheres with moderate Reynolds numbers," Phys. Fluids 19, 093302 (2007).

${ }^{15}$ S. Y. Tee, P. J. Mucha, M. P. Brenner, and D. A. Weitz, "Velocity fluctuations in a low-Reynolds-number fluidized bed," J. Fluid Mech. 596, 467 (2008).

${ }^{16}$ J. F. Richardson and W. N. Zaki, "Sedimentation and fluidization: Part I," Trans. Inst. Chem. Eng. 32, 35 (1954).

${ }^{17}$ P. Mills and P. Snabre, "Settling of a suspension of hard spheres," Europhys. Lett. 25, 651 (1994).

${ }^{18} \mathrm{P}$. Snabre and P. Mills, "Settling and fluidization of non Brownian hard spheres in a viscous liquid," Eur. Phys. J. E 1, 105 (2000).

${ }^{19}$ J. Kozeny, "Ueber kapillare leitung des wassers im boden," Ber Wien Akad 136a, 271 (1927).

${ }^{20}$ P. C. Carman, "Fluid flow through granular bed," Trans. Inst. Chem. Eng. 15, 150 (1937); "Some physical aspects of water flow in porous media," Discuss. Faraday Soc. 3, 72 (1948).

${ }^{21}$ N. Péron, S. J. Cox, S. Hutzler, and D. Weaire, "Steady drainage in emulsions: Corrections for surface Plateau borders and a model for high aqueous volume fraction," Eur. Phys. J. E 22, 341 (2007).

${ }^{22}$ G. K. Batchelor, "Sedimentation in a dilute dispersion of spheres," J. Fluid Mech. 52, 245 (1972).

${ }^{23}$ R. E. Caflisch and J. H. C. Luke, "Variance in the sedimentation speed of a suspension," Phys. Fluids 28, 759 (1985); M. P. Brenner, "Screening mechanisms in sedimentation," ibid. 11, 754 (1999); X. Yin and D. L. Koch, "Velocity fluctuations and hydrodynamic diffusion in finiteReynolds-number sedimenting suspensions," ibid. 20, 043305 (2008). 